\title{
MODERN STEREOLOGICAL EVALUATION IN THE AGING HUMAN SUBSTANTIA NIGRA
}

\author{
${ }^{1}$ ShUANG Y MA, ${ }^{1}$ FranK M LONGO, ${ }^{2}$ MATIAS RÖYTTÄ AND ${ }^{2}$ YrJÖ COLLAN
}

${ }^{1}$ Department of Neurology, School of Medicine, University of North Carolina, CB 7025, Chapel Hill, North Carolina, 27599, USA; ${ }^{2}$ Department of Pathology, University of Turku, Kiinamyllynkatu 10, FIN-20520, Turku, Finland e-mail: mas@glial.med.unc.edu (Accepted June 6, 2003)

\begin{abstract}
Quantitative estimation of neuronal numbers in the human substantia nigra (SN) can be achieved by a conventional single section (SS) count or by the more modern stereological disector (DS) count. However, counting results from SS counts are potentially biased and might not accurately reflect the total neuronal number in the $\mathrm{SN}$ or the changes in the total number of neurons occurring during aging or with neurodegenerative disease. Potential sources of bias include the lack of linearity between cell number per area of section and cell number per volume; the variation in the counting level and orientation of tissue sections; and shrinkage of tissue. Modern stereological DS counting overcomes these problems and has played a crucial role in many recent studies in neuropathology, neuroanatomy, neuropharmacology and neurogenetics. Over the past decades, four stereology based counting methods including physical DS, physical fractionator, optical DS and optical fractionator, have been established for quantitative measurement. Recently, stereological estimates have revealed a linear reduction rate of total nigral neuronal numbers with age of about $10 \%$ per decade. These findings suggest that the surviving nigral neurons undergo a degenerative change leading to neuronal dysfunction with aging. Furthermore, as an advanced quantitative tool, modern stereological evaluation may provide new insights into the aging of the human SN thereby enabling us to better understand the pathophysiological processes in aging brain.
\end{abstract}

Keywords: aging, quantitation, stereology, substantia nigra.

\section{INTRODUCTION}

Quantitative estimation using modern stereological disector (DS) counts combined with Cavalier's principle plays a crucial role in neuropathological, neuroanatomical, neurogenetic and neuromorphological studies (Ma et al., 1999a; b; Long et al., 1999; Dowd et al., 2000; Stein et al., 2000; Greferath et al., 2000). The modern stereological technique has been used extensively to estimate changes in the total number of neurons in various brain nuclei associated with aging (Ma et al., 1999a, b; Ma 1997; Park et al., 2001; Perl et al., 2000; West 1993a). Recently, quantitative studies using DS counts have revealed a selective age-related neuronal loss in vulnerable brain nuclei, particularly in the human substantia nigra (SN) (Ma et al., 1999a, b). Previous studies using traditional single section (SS) counts in aging human SN have also demonstrated a similar decrease of nigral neurons with advancing age (Hirai, 1968; McGeer et al., 1977; Mann and Yates, 1984; Fearnley and Lee, 1991).
Although quantitative counting methods in the aging human SN can apply either SS or DS counts, the accuracy of the counting method plays an important role in the overall impact of quantitative studies in aging human SN research (Saper 1996; Guillery and Herrup 1997; Williams and Pakic 1988). In this review, we will address principles of stereology, application of neurostereology, and the findings revealed by quantitative measurement in the aging human $\mathrm{SN}$.

\section{SINGLE SECTION COUNTS IN THE AGING HUMAN SUBSTANTIA NIGRA}

There is a long history of traditional estimation of neuronal number using SS counts in quantitative studies of the aging human SN (Hirai, 1968; McGeer et al., 1977; Mann and Yates, 1982; Fearnley and Lee, 1991). These quantitative evaluations may be associated with considerable variation in application and therefore may underestimate or overestimate nigral neuronal number (Hirai, 1968; McGeer et al., 1977; Mann and Yates, 1982; Fearnley and Lee, 
1991; Pakkenberg et al., 1991; Ma et al., 1997, 1999a, b). Multiple factors can affect the number of nigral neurons estimated from SS counts in the aging human $\mathrm{SN}$, including counting levels, section orientation, and tissue shrinkage.

\section{COUNTING LEVELS IN THE SUBSTANTIA NIGRA}

The SN, a small and irregular nucleus, is located in the brain stem and contains pigmented neuromelanin cells (Olszewski and Baxter, 1964; van Domburg et al., 1992). According to anatomical topography, the SN is composed of various irregular sub-nuclei, such that nigral neurons are not evenly distributed along the rostral-caudal axis within the SN (Olszewski and Baxter, 1964; Ma et al., 1995a, b; 1997). Therefore, the selected level of examination may be an important factor in neuronal counts. In most SS-based studies estimating the number of neurons in the SN, only one level across the $\mathrm{SN}$ is assessed (Fearnley and Lee, 1990; German et al., 1995; Ma et al., 1995a, b). These studies using SS counts from various regions of the $\mathrm{SN}$ have revealed that the greatest number of nigral neurons appears at the middle portion of the SN. Both the far rostral and the far caudal portion of the SN have much smaller numbers of neurons (Ma et al., 1995a, b; Ma, 1997). In this regard, the variation of nigral neuronal counts between the middle and far rostral or far caudal portions is considerable (Hirai, 1968; McGeer et al., 1977; Mann and Yates, 1982; 1984; Fearnley and Lee, 1991; German et al., 1989; Ma et al., 1995a, b; 1996; Ma, 1997). As illustrated in Fig. 1, if the counting level is not perfectly matched with the selected level in the mid-portion of SN, or with a predefined level, one may obtain a large variation in the nigral neuronal count. Another source of variation is that cell numbers estimated from SS counts are dependent on the outlined area of estimation. Generally, in SS counts in the aging human SN the goal has been to obtain a section from the midportion of the SN, e. g., at the level of exit of the third cranial nerve or the level of the superior colliculus and caudal part of the red nucleus (Hirai, 1968; McGeer et al., 1977; Mann and Yates, 1982; German et al., 1989; Fearnley and Lee, 1991; Ma et al., 1995a, b; Ma, 1997). These levels of cross sections provide a large area for quantitative estimation in SS counts. However, in practice, it is difficult to validate the actual level of the section obtained for analysis. Consequently, the results from different levels of the SN cannot reliably be compared with each other in the aging human SN. Thus, results from quantitative analyses using SS counts cannot be easily compared between laboratories, and the results must be interpreted with caution in terms of quantitative estimation of total numbers of neurons (McRitchie et al., 1996).

$$
\begin{array}{lll}
\text { Case } & \text { A } & \text { B }
\end{array}
$$

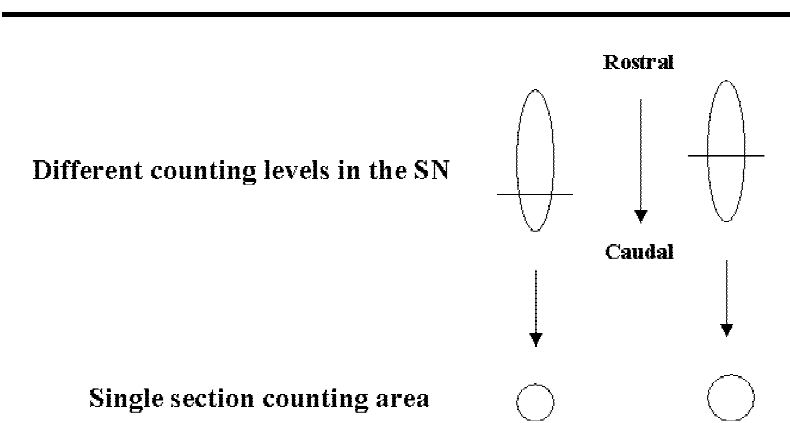

Fig. 1. Different counting levels in the human $S N$ are illustrated. If the counting level (case A) is not accurately and consistently located at the same counting level (case B) in the mid-portion of the $S N$ or at some other with-defined levels, a large variation in estimates from SS counts will result. Here, case A will estimate less nigral neurons than case $B$ because case $B$ has a larger counting region.

\section{ORIENTATIONS OF TISSUE SECTIONS}

There are several ways to remove the brain stem and to sample tissue blocks containing the $\mathrm{SN}$, such as cutting perpendicular (coronal sections) or parallel (sagital sections) from the long axis of the brain stem (Ma et al., 1995a, b; McRitchie et al., 1996; Ma, 1997). Potential variations in orientation of brain tissue sections are illustrated in Fig. 2. Consistent with the large counting region in case B compared to case A (Fig. 2), it has been clearly demonstrated that increased areas lead to an increase in the number of cells counted.

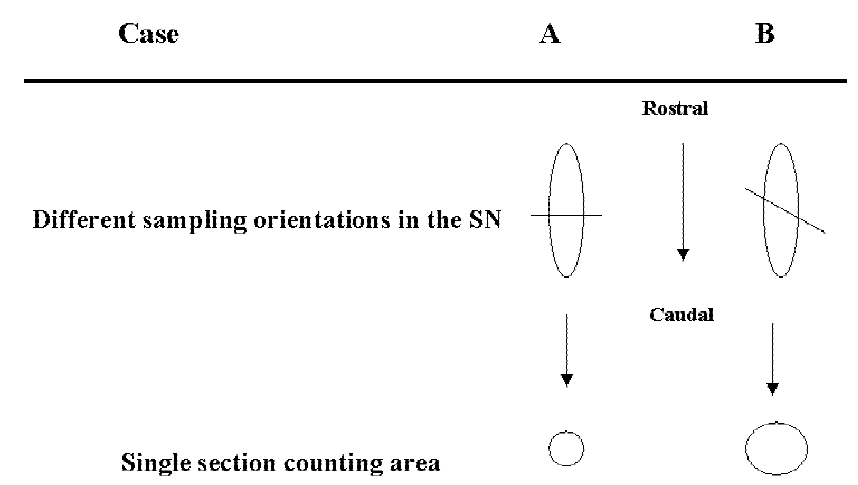

Fig. 2. This figure reveals different orientations of tissue sections in the human SN. If the orientation of tissue section is not closely defined and followed, one will obtain a considerable variation in SS counts. In this case, sample B will lead to detection of more nigral neurons than that of sample A. 


\section{TISSUE SHRINKAGE IN THE SUBSTANTIA NIGRA}

Tissue shrinkage may be one of variables affecting the results estimated from SS counts. After its removal from the brain stem, the tissue containing SN is usually fixed in $4 \%$ phosphate-buffered formalin, which may cause primary tissue shrinkage. Following that, the tissue blocks are processed for paraffin embedding or saturated with $30 \%$ sucrose. Based on different immunostaining and fixation techniques, tissue shrinkage may occur after the immunohistological staining processes (Ma et al., 1999b). Along with tissue shrinkage, neurons also undergo shrinkage. If only a single section is counted, the reduction in neuronal size would lead to failure to detect some neurons and lead to an underestimate of neuronal number. The conventional SS counting method is a biased two-dimensional sampling approach, and the problem is that the number of neurons detected per area of tissue is a more complicated feature than the number of neurons actually present per volume of tissue. In addition to variations in counting level, in tissue shrinkage and in orientation of the sample in SS counts, the number of neuronal profiles detected per unit area in twodimensional sections is also influenced by other factors, such as section thickness, the average diameter of neurons, the density of neurons, truncation of nuclei and the percentage of lost caps (truncation) (Ma, 1997, Dorph-Petersen et al., 2001). Furthermore, the number of neuronal profiles per unit area in twodimensional sections may also vary due to anisotropy in the SN. Taken altogether, SS estimates may produce large variations of nigral neuronal counts in the human SN studies (Hirai, 1968; McGeer et al., 1977; Mann and Yates, 1982; Fearnley and Lee, 1991). This large variability and underestimation or overestimation may be related to biological differences, variable counting levels, different orientations of sampling tissue sections and also tissue shrinkage (Ma et al., 1995a, b; 1996; Ma, 1997). Therefore, in terms of quantitative analysis, the results from SS counts may not necessarily be reliably comparable across different studies. In addition, SS counts cannot be used to reliably estimate the absolute number of neurons.

\section{COMPARISON OF SS AND DS COUNTING METHODS}

SS-based counting method assumes that cell density is uniform throughout the SN. In the DS method, application of Cavalier's principle allows the estimation of the total number of neurons in the whole SN regardless of its size, shape or orientation (Sterio, 1984; Pakkenberg et al., 1988; 1991; West, 1993b; Ma, 1997; Ma et al., 1999a, b). Methodological differences between SS and DS counting systems are summarized in Table 1. The SS approach measures the number of neurons per area in one section of the $\mathrm{SN}$ (neurons $/ \mathrm{mm}^{2}$ ), whereas the DS approach counts neurons in consequative sections and estimates the number of neurons per volume (neurons $/ \mathrm{mm}^{3}$ ). The cutting of sections for the SS counts is simple, but cutting of sections for the DS counts is laborious, and takes more time (Collan et al., 1984; 1983; 1991; 1992; Ma, 1997; Ma et al., 1995a, b; 1999a, b). In terms of topography of neuroanatomic structures and neuromorphological evaluation, DS counts with Cavalieri's principle of volumetric measurement provide more information for quantitative estimations and for morphological structures, such as the volume of brain nuclei, and cell density within nuclei compared to SS-based counts. In quantitative evaluations in aging brain, stereological counting methods offer the advantages of reproducible and stereologically unbiased analyses. Furthermore, Cavalieri's principle and the methodology of DSbased assessment can be learned after a reasonably short period of training.

Table 1. Comparison of single section and disector counts.

\begin{tabular}{lll}
\hline & Single section counts & Disector counts \\
\hline Brain tissue & one block & many blocks \\
Sections & one section & many sections \\
Neuronal density & neurons $/ \mathrm{mm}^{2}$ & neurons $/ \mathrm{mm}^{3}$ \\
Measurement & two-dimensional & three-dimensional \\
Counted neurons & at one sectional level (location) & serial sections from the whole nucleus \\
Time consumption & modest & high \\
Accuracy & uncertain / biased & more certain / unbiased \\
Variation sources: & counting level & not dependent on single level \\
& sampling orientation & not affected by section orientation \\
& thickness of section & the same section thickness \\
& shrinkage of tissue & shrinkage may or may not affect the result \\
\hline
\end{tabular}




\section{PRINCIPLE OF STEREOLOGICAL EVALUATION IN THE SUBSTANTIA NIGRA}

\section{Tissue preparation}

To establish a stereology based counting method for assessment of the SN tissue blocks harvested from human brain should be well-defined and include the entire SN, and a well-defined brain tissue block (Ma et al., 1999a, b). Tissue preparations for sampling, fixation and storage should be taken in a uniform, systematic and unbiased manner in order to reduce sampling variations (Ma, 1997; Ma et al., 1999a, b).

\section{Penetration assessment of immunostaining}

For an unbiased and accurate stereological results from immunohistological investigation, one must confirm uniform penetration of immunostaining throughout the entire thickness of the tissue section. Such evaluation can be done with scanning at $1-$ $5 \mu \mathrm{m}$ intervals along the z-axis (i.e., vertical axis) through the whole tissue section from the upper surface to the bottom of tissue section. If the whole tissue section is not totally penetrated by antibody; one will underestimate the immunohistochemical positive neurons. In applying immunostaining for tyrosine hydroxylase (TH) or the dopamine transporter (DAT), a floating section thickness of $40 \mu \mathrm{m}$ allows adequate tissue penetration and immuno-visualization (Ma et al., 1999b).

\section{Criteria for neuronal counts}

Counting of neurons with a stereologically unbiased procedure requires careful planning for the sampling procedure. The counting fields should be randomly chosen and systematically scanned. To avoid duplicate estimation, a counting frame should be always applied (Gundersen, 1978). In the ideal case, counts are based on identification of nuclei if they are clearly visualized by immunostaining. In cases in which nuclei are not well visualized neuronal bodies are counted (Ma, 1997; Ma et al., 1999a, b). The neuronal profiles or the nuclei should be clearly focused. With high neuronal densities, one should be careful in estimating the defined counting height $(\mathrm{h})$ to avoid overlapping of counts. The designation of counting height is based on the diameter of nucleus or diameter of neuron. Generally, the counting height should be greater than the diameter of nuclei or diameter of neurons counted. Fig. 3 demonstrates how to determine the counting height (h) using either the neuronal body or neuronal nucleus in the entire tissue section. As the picture illustrates, both surface and bottom of tissue regions are regarded as guard zones or forbidden areas for neuronal estimates because of the uneven tissue levels. Furthermore, to obtain an unbiased and accurate result from DS estimation, counts should be performed in a blinded manner.

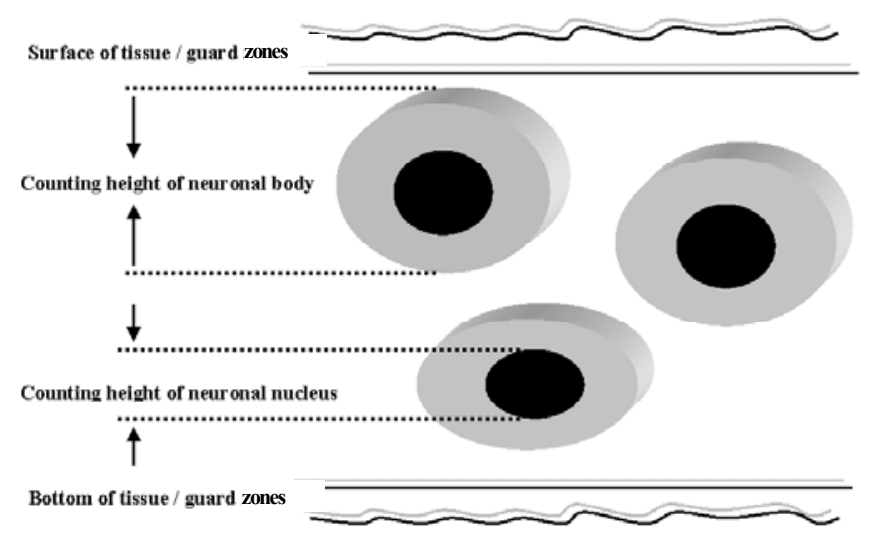

Fig. 3. Demonstrates determination of counting height (h) using either the neuronal body or neuronal nucleus in the tissue section. As illustrated, both regions of the surface and bottom of tissue are regarded as a guard zones in which neurons are not counted because of uneven tissue topography.

\section{Assessment of variance in neuronal counts}

For quantitative studies, assessment of variance is important to assure an unbiased result. There are two major sources of variations including application of quantitative methods and intrinsic biological differences between samples. However, in order to reduce variations from application of counting methods, principles of stereology, systematic tissue preparation, systematic random sampling, confirmation of penetration of immunostaining; and consistent application of wellestablished criteria for neuronal counts should be applied. To minimize biological variations, one should use age / sex - matched and homologous animal samples (Gundersen and Østerby, 1981; Gundersen and Jensen, 1987; Tang, 1999).

\section{COMPARISON OF PHYSICAL / OPTICAL DISECTOR AND PHYSICAL / OPTICAL FRACTIONATOR COUNT}

Applied with the Cavalier's principle, currently there are four different stereologically valid counting methods. Using physical disector and physical fractionator approaches, two adjacent sections are examined and 
neurons only present in the sampling section, but not in the adjacent look-up section, should be counted. In optical disector and optical fractionator counts a computer-based automatic stage allows to optical scanning and counting throughout serial tissue sections or continual optical dissections within a given section. The advantages and disadvantages of these methods have been discussed (Braendgaard and Gundersen 1986; Dorph-Petersen et al., 2001). The physical DS approach is used for counting small structures such as neurons, synapses, fibers and axons, under an electron microscope or a light microscope with a high magnification using a pair of adjacent sections or serial sections (Collan et al., 1992; Ma et al., 1995a, b; Ma, 1997; Tang et al., 2001). Tissue preparation and neuronal counts incorporating matching neurons in pairs of sections in physical DS and in serial sections in physical fractionator counts may take more time than that required for optical dissector or optical fractionator studies. Matching of neurons in paired sections is not always possible in the physical DS or physical fractionator counts, but is necessary. If reliable results are to be obtained with high numerical densities, physical DS and physical fractionator methods are more likely to produce variation resulting from the overlapping of objects. Section thickness for physical DS and physical fractionator counts should be $\leq 5 \mu \mathrm{m}$, whereas section thickness for optical DS and optical fractionator counts should be greater $(\geq 25 \mu \mathrm{m})$. With the availability of computer-based visualization approaches, the optical DS and the optical fractionator counts have become more frequently applied. Computer-based control of an automatic stage allows optical dissection along the $Z$-axis of the tissue section. Optical dissection throughout the Z-axis allows assessment of neurons throughout the entire thickness of the three-dimensional tissue section and thus allows a greater number of images to be assessed than that allowed using physical DS or physical fractionator approaches. The computerized stereological evaluation allows efficient, consistent and accurate estimate of the total number of neurons from the entire targeted nucleus. Estimates for total number of nigral neurons using physical fractionator counts is not dependent on volume measurement of the whole SN, and is not affected by tissue shrinkage (Ma, 1997; Dorph-Petersen et al., 2001). Table 2 compares physical DS / fractionator and optical DS / fractionator counts.

\section{QUANTITATIVE EVALUATION IN THE AGING HUMAN SUBSTANTIA NIGRA}

The relationship between normal aging and decline in the number of dopaminergic neurons in the $\mathrm{SN}$ was reported by a study using SS counts several decades ago (Hirai, 1968). A subsequent investigation using SS counts demonstrated a decrease in SN neurons with advancing age in the human $\mathrm{SN}$ at a rate of $4.7 \%$ per decade (Fearnley and Lee, 1990). Several lines of evidence have revealed that age-related neuronal loss occurs at significantly different rates in different brain nuclei (West 1993a; Ma et al., 1999a, b). Application of DS-based counts using principles of stereology with Cavalieri's principle has confirmed a linear relationship between increasing age and decreasing neuron number. Stereological evaluation has revealed decreases in neuronal numbers at a rate of $9.8 \%$ per decade from age 17 to 90 years. In addition to the nigral neuronal loss, the average nigral neuronal size decreases by $3.2 \%$ per decade, suggesting that the surviving nigral neurons are under an age-related neuronal degenerative process (Ma et al., 1999a). Recent studies using DAT and guanosine triphosphate cyclohydrolase I (GTPCHI) immunostaining have demonstrated a decrease of $88 \%$ in counts of DAT-staining neurons and a decrease of $81.5 \%$ in counts of GTPCHI-staining neurons within the $\mathrm{SN}$ in elderly individuals as compared to young individuals (Ma et al., 1999b; Chen et al., 2000).

Table 2. Comparison of physical disector/fractionator and optical disctor/fractionator.

\begin{tabular}{lll}
\hline & Physical disector / fractionator & Optical disector / fractionator \\
\hline Microscopes & two & one \\
Imaging evaluation & two images & one with section height \\
Time consumption & high & modest \\
Tissue thickness & thinner $(\leq 5 \mu \mathrm{m})$ & thicker $(\geq 25 \mu \mathrm{m})$ \\
Sample volume/space & measurement $/$ regardless of space & measurement $/$ regardless of space \\
Counting sections & \multicolumn{1}{c}{ the sections are systematically selected (i.e., every third) from a set of serial sections } \\
Neuronal density & neurons $/ \mathrm{mm}^{3} /$ irrelevant neuronal density & neurons $/ \mathrm{mm}^{3} /$ irrelevant neuronal density \\
Mathematic formula & $\mathrm{N}=\mathrm{N}_{\mathrm{V}} \cdot \mathrm{V} / \mathrm{N}=\sum \mathrm{Q} \cdot \mathrm{t} / \mathrm{h} \cdot 1 / \mathrm{asf} \cdot 1 / \mathrm{ssf}$ & $\mathrm{N}=\mathrm{N}_{\mathrm{V}} \cdot \mathrm{V} / \mathrm{N}=\sum \mathrm{Q} \cdot \mathrm{t} / \mathrm{h} \cdot 1 / \mathrm{asf} \cdot 1 / \mathrm{ssf}$ \\
\hline
\end{tabular}

$\mathrm{N}=$ total number; $\mathrm{N}_{\mathrm{V}}=$ density; $\mathrm{V}=$ volume; $\Sigma \mathrm{Q}=$ sum of disector numbers; $\mathrm{t}=$ thickness; $\mathrm{h}$ = height; asf = areal sampling fraction and $\mathrm{ssf}=$ section-sampling fraction 
Table 3. Quantitative estimates in the aging human substantia nigra.

\begin{tabular}{llll}
\hline Studies & Number & $\begin{array}{l}\text { Percentage } \\
\text { Density }\left(\text { neurons } / \mathrm{mm}^{3}\right)\end{array}$ & $\begin{array}{l}\text { reduction with age } \\
\text { Volume }\left(\mathrm{mm}^{3}\right)\end{array}$ \\
\hline SS Counts: & & & \\
\hline Hirai, 1968 & 38 (elderly vs. young) & n.a. & n.a. \\
McGeer et al., 1977 & 48 (elderly vs. young & n.a. & n.a. \\
Mann et al., 1984 & 36 (elderly vs. young) & n.a. & n.a. \\
Fearnley and Lee, 1991 & 4.7 (per decade) & n.a. & n.a. \\
\hline DS Counts: & & & 4.4 \\
Ma et al., 1999a & 9.8 (per decade) & 7.4 & 21 \\
Ma et al., 1999b & 88 (elderly vs. young) & 85 & n.a. \\
Chen et al., 2000 & 81.5 (elderly vs. young) & 81.8 & \\
\hline
\end{tabular}

n.a. $=$ not available and SS counts $=$ number of neurons per section, *type I DAT neurons

These studies using stereological evaluations confirm the previous studies using SS counts demonstrating a decline of nigral neuronal numbers with aging. Table 3 compares quantitative estimates using SS and DS counts in the human aging SN. The percentage reduction of nigral neurons per decade with age derived from DS counts is more than double that derived from SS counts, indicating that previous investigations using SS counts underestimated the extent of age-related nigral neuronal loss (Hirai, 1968; McGeer et al., 1977; Fearnley and Lee, 1990; Ma et al., 1999a, b; Chen et al., 2000). Therefore, three-dimensional quantitative measurements provide a more accurate insight into the pathophysiological alterations in the aging human SN.

\section{CONCLUSIONS}

Age-related nigral neuronal loss has been well documented by studies using either SS or DS counts. Modern stereological DS methods estimating the total number of nigral neurons have had a significant impact on elucidating the effects of aging on the human SN. Stereological counts have become a reliable, accurate and reproducible method, which is not affected by the counting level, orientation of the sample, or tissue shrinkage in the aging human SN. Studies of neuronal numbers should incorporate the principles of stereology, uniform tissue preparation, systematic random sampling, confirmation of immunostaining peneration, well-defined criteria for neuronal counts, and evaluation of variance.

\section{REFERENCES}

Abercrombie M (1946). Estimation of nucleus population from microtome sections. Anat Rec 94:239-47.

Braendgaard H, Evans SM, Howard CV, Gundersen HJG (1990). The total number of neurons in the human neocortex unbiasedly estimated using optical disectors. J Microsc 157:285-304.
Braendgaard H, Gundersen HJG (1986). The impact of recent stereological advances on quantitative studies of the nervous system. J Neuroscience Methods 18:39-78.

Cavalieri B (1966). Geometria degli indivisibili. Torino: Unione Tipografico, Editrice, pp. 1-543.

Chen EY, Kallwitz E, Leff SE, Cochran FJ, Mufson EJ, Kordower JH (2000). Age - related decreas in GTPcychlohydrolase-I immunoreactive neurons in the money and human substantia nigra. J Comp Neurol 426(4):534-48.

Coggeshall RE (1992). A consideration of neural counting methods. Trends Neurosci 15:9-13.

Collan Y (1991). Quantitative histopathology of soft tissue clacifications. Proc Finn Dent Soc 87:643-50.

Collan Y, Aalto ML, Kosma VM (1984). Stereology and morphometry in pathology. Kuopio: Kuopio University Press, 1-270.

Collan Y, Oja E, Whimster W (1983). Mathematical background to stereology and morphometry for diagnostic pathologists. Acta Stereol 2:214-38.

Collan Y, Ma SY, Röyttä M, Rinne JO, Rinne UK (1992). Experience on the use of the disector principle in neuropathology. Acta Stereol 11(1):52-62.

Cruz-Orive LM, Weibel ER (1990). Recent stereological methods for cell biology: a brief survey. Am J Physiol 258 (Lung Cell Mol Physiol 2):L148-56.

Dowd G, Counia U, Ma SY, Kehr Jan Bacon A, Allen S, Holmes F, Wang FH, Mufson EJ, Davies C, Dawson G, Wynick D (2000). Targeted disruption of the galanin gene reduces the number of basal forebrain cholinergic neurons and impairs learning and longterm potential in an age-dependent manner. Proc Natl Acad Sci 97(21):11569-74.

Dorph-Petersen KA, Nyengaard JR, Gundersen HJ (2001). Tissue shrinkage and unbiased stereological estimation of particle number and size. J Microsc 204(3):232-46.

Fearnley JM, Lees AJ (1991). Ageing and Parkinson's disease: Substantia nigra regional selectivity. Brain 114:2283-301.

German DC, Manaye K, Smith WK, Woodward DJ, Saper CB (1989). Midbrain dopaminergic cell loss in 
Parkinson's disease: computer visualization. Ann Neurol 26:507-14.

Guillery RW and Herrup R (1997). Quantification without poinfication: choosing a method for counting objects in sectioned tissue. J Comp Neurol 386:2-7

Gundersen HJ (1978). Estimators of the number of objects per area unbiased by edge effects. Microsc Acta 81(2):107-17.

Gundersen HJ, Østerby R (1981). Optimizing sampling efficiency of stereological studies in biology: or' do more less well! J Microsc 121(1):65-73.

Gundersen HJG (1986). Stereology of arbitrary particles: A review of unbiased number and size estimators and the presentation of some new ones, in memory of William R. Thompson. J Microsc 143:3-45.

Gundersen HJ, Jensen EB (1987). The efficiency of systematic sampling in stereology and its prediction. J Microsc 147(3):229-63.

Gundersen HJG, Bagger P, Bendtsen TF, Evans SM, Korbo L, Marcussen N, Møller A, Nielsen K, Nyengaard JR, Pakkenberg B, Sørensen FB, Vesterby A, West MJ (1988). The new stereological tools: disector, fractionator, nucleator and point sampled intercepts and their use in pathological research and diagnosis. APMIS 96:857-81.

Gundersen HJG, Jensen EB (1987). The efficiency of systematic sampling in stereology and its prediction. J Microsc 147:229-63.

Hirai S (1968). Ageing of the substantia nigra. Adv Neurol Sci $12845-9$.

Long JM, Mouton PR, Jucker M, Ingram DK (1999). What counts in brain aging? Design-based stereological analysis of cell number. J Gerontol A Biol Sci Med Sci 54A(10):B401-17.

Mann DMA, Yates PO, Marcyniuk B (1984). Monoaminergic neurotransmitter systems in presenile Alzheimer's disease and in senile dementia of Alzheimer type. Clin Neuropathol 3:199-205.

Mann DMA, Yates PO (1982). Pathogenesis of Parkinson's disease. Arch Neurol 39:545-9.

McRitchie DA, Halliday GM, Pamphlett R (1996). Diagnostic evaluation of the substantia nigra. Neuropathol Appl Neurobiol 22:228-32.

Ma SY (1997). The substantia nigra in Parkinson's disease: a morphological study on neuronal changes. Academical thesis, University of Turku, Finland.

Ma SY, Röyttä M, Rinne JO, Collan Y, Rinne UK (1995a). Single section and disector counts in evaluating neuronal loss in the substantia nigra in patients with Parkinson's disease. Neuropathol Appl Neurobiol 21:341-3

Ma SY, Röyttä M, Rinne JO, Collan Y, Rinne UK (1995b). Cell counts in the substantia nigra: A comparison of single section count and disector counts in patients with Parkinson's disease and in normal controls. Neuropathol Appl Neurobiol 21(1):10-17.

Ma SY, Rinne JO, Röyttä M, Collan Y, Rinne UK (1997).
Correlation between neuromorphometry in the substantia nigra and clinical features in Parkinson's disease using disector counts. J Neurol Sci 15:83-7.

Ma SY, Rinne JO, Collan Y, Röyttä M, Rinne UK (1996). A quantitative morphometrical study of the neurodegeneration in patients with Parkinson's disease. J Neurol Sci 140:40-5.

Ma SY, Röyttä M, Collan Y, Rinne JO (1999). Unbiased morphometrical measurements show nigral neuronal loss with aging. Neuropathol Appl Neurobiol 25:394-9.

Ma SY, Jaffar S, Kordower JH, Ciliax JJ, Cochran E, Mash DC, Mufson EJ (1999). Dopamine transporterimmunoreactive neurons decrease with age in the human substantia nigra. J Comp Neurol 409:25-37.

Mayhew TM (1992). A review of recent advances in stereology for quantifying neural structure. J Neurocytol 21:313-28.

McGeer PL, McGeer EG, Suzuki JS (1977). Aging and extrapyramidal function. Arch Neurol 34: 33-5.

Olszewski J and Baxter D (1964). Cytoarchitechitecture of the human brain stem. Basel: Karger, pp. 131.

Pakkenberg B, Gundersen HJG (1988). Total number of neurons and glial cells in human brain nuclei estimated by the disector and the fractionator. J Microsc 150:1-20.

Pakkenberg B, Møller A, Gundersen HJG, Dam AM, Pakkenberg H (1991). The absolute number of nerve cells in substantia nigra in normal subjects and in patients with Parkinson's disease estimated with an unbiased stereological method. J Neurol Neurosurg Psychiat 54:30-3.

Park JJ, Tang Y, Lopez I, Ishiyama A (2001). Age-related change in the number of neurons in the human vestibular ganglion. J Comp Neurol 19:431(4):437-43.

Perl DP, Good PF, Bussiere T, Morrison JH, Erwin JM, Hof PR (2000). Practical approaches to stereologyv in the setting of aging- and disease-related brain banks. J Chem Neuroanat 20(1):7-19.

Schmitz C, Hof PR (2000). Recommendations for straightforward and rigorous methods of counting neurons based on a computer simulation approach. J Chem Neuroanat 20(1):93-114

Sender T, Ma SY, Jaffar S, Kordower JH, Mufson EJ (2000). Total trkA neuronal loss in the nucleus basalis in Down's syndrome: an unbiased stereological count. J Neurochem 74:1185-96.

Steiner RA, Hohmann JG, Holmes A, Cadd G, Jureus A, Clifton DK, Luo Mulon, Gustshall M, Ma SY, Mufson EJ, Crawley JN (2001). Memory deficits in mice overexpressing galanin. Proc Natl Acad Sci USA 27:98(7):4184-9.

Sterio DC (1984). The unbiased estimation of number and sizes of arbitrary particles using the disector. $J$ Microsc 134:127-36.

van Domburg PHMF, ten Donkelaar HJ (1992). The human substantia nigra and ventral tegmental area: A neuroanatomical study with notes on aging and aging 
disease. Adv Anat Embryol Cell Biol 121:32-69.

Weibel ER (1979). Stereological methods. Practical methods for biological morphometry. London: Academic Press, vol. 1, 1-415.

Williams RW, Pakic P (1988). Three-Dimensional counting: an accurate and direct method to estimate numbers of cells in sectioned material. J Comp Neurol 278:344-52.

West MJ (1993a). Regionally specific loss of neurons in the aging human hippocampus. Neurobiol Aging $14: 287-93$.
West MJ (1993b). New stereological methods for counting neurons. Neurobiol Aging 14:275-85.

Tang Y (1999). A stereological study of neuronal connections (myelinated fibers of white matter and synapses of neocortex) in the human brain. Academical thesis, University of Aarhus, Denmark.

Tang Y, Jens R, Nyengaard, Didima MG, De Groot, Gundersen HG (2001). Total regional and global number of synapses in the human brain neocortex. Synapse 41:258-73. 